\title{
SIGNED GRAPHS WITH TOTALLY DISCONNECTED STAR COMPLEMENTS
}

\author{
ZORAN STANIĆ
}

\begin{abstract}
We are interested in a signed graph $\dot{G}$ which admits a decomposition into a totally disconnected (i.e., without edges) star complement and a signed graph $\dot{S}$ induced by the star set. In this study we derive certain properties of $\dot{G}$; for example, we prove that the number of (distinct) eigenvalues of $\dot{S}$ does not exceed the number of those of $\dot{G}$. Some particular cases are also considered.
\end{abstract}

\section{INTRODUCTION}

Given a graph $G=(V(G), E(G))$, let $\sigma: E(G) \rightarrow\{1,-1\}$. Then $\dot{G}=(G, \sigma)$ is a signed graph derived from its underlying graph $G$. To ease language, we say that each edge $e \in E(G)$ is accompanied by the sign $\sigma(e) \in\{1,-1\}$. The (multiplicative) group $\{1,-1\}$ can also be written as $\{+,-\}$. We denote $|V(\dot{G})|$ by $n$. The edge set of a signed graph is composed of subsets containing positive and negative edges, respectively. Throughout the paper, we interpret an (unsigned) graph as a signed graph with all the edges being positive.

The adjacency matrix $A_{\dot{G}}$ of $\dot{G}$ is obtained from the $(0,1)$-adjacency matrix of $G$ by reversing the sign of all 1 s which correspond to negative edges. The eigenvalues of $A_{\dot{G}}$, together with their repetition, form the spectrum of $\dot{G}$.

We recall from [3, 6] the existence of a spectral tool (known as a star complement technique) for constructing comparatively large graphs with specified spectral properties from their smaller parts, known as star complements; these can be defined in the case of signed graphs as well (the definition is given in the next section). This motivates us to consider signed graphs which admit a vertex set partition into two non-empty subsets such that the subgraph induced by one of these subsets is a particular (totally disconnected) star complement in $\dot{G}$. If $\dot{G}$ admits the mentioned partition, then we briefly say that $\dot{G}$ can be decomposed into the corresponding

2020 Mathematics Subject Classification. 05C22, 05 C50.

Key words and phrases. Signed graph decomposition; adjacency matrix; star set; signed line graph; exceptional signed graph.

Research is partially supported by the Serbian Ministry of Education, Science and Technological Development via the Faculty of Mathematics, University of Belgrade. 
subgraphs. We derive certain properties of such signed graphs and their star complements, and consider some particular cases. Similar decompositions of graphs can be found in [5, 10].

\section{Preliminaries}

Our terminology and notation are transferred from the domain of graphs - see [2, 3, 8, for example, we say that a signed graph is connected, regular, or bipartite if the same holds for its underlying graph. We use $K_{n}$ to denote the complete graph with $n$ vertices and $k K_{n}$ for the union of $k$ disjoint copies of $K_{n}$. In particular, $n K_{1}$ denotes the totally disconnected (i.e., without edges) graph with $n$ vertices. The all-1, the all-0, and the identity matrices are denoted by $J, O$, and $I$, respectively. To avoid possible confusion, their size will occasionally be given as a subscript.

If the vertices $i$ and $j$ are adjacent (i.e., if there is either a positive or a negative edge between them), we write $i \sim j$. In particular, $i \stackrel{ \pm}{\sim} j$ (resp. $i \succsim j$ ) designates the existence of a positive (resp. negative) edge between $i$ and $j$.

The graphs $\dot{G}$ and $\dot{H}$ are said to be switching equivalent if there exists a set $U \subseteq V(\dot{G})$ such that $\dot{H}$ is obtained from $\dot{G}$ by reversing the sign of every edge with exactly one end in $U$. Switching equivalent signed graphs share the same underlying graph and the same spectrum. Equivalently, if the vertex labelling of $\dot{G}$ and $\dot{H}$ is transferred from their common underlying graph, then they are switching equivalent if there exists a diagonal matrix $D$ of \pm 1 s such that $A_{\dot{H}}=D^{-1} A_{\dot{G}} D$. We say that $\dot{G}$ and $\dot{H}$ are switching isomorphic if one of them is isomorphic to a signed graph which is switching equivalent to the other one. Switching isomorphic signed graphs are usually identified.

A cycle in a signed graph is said to be positive if the number of negative edges contained is not odd. Otherwise, it is said to be negative.

A signed doubled graph $\ddot{G}$ is obtained by inserting a negative (parallel) edge between every pair of adjacent vertices of a graph $G$; precisely, $\ddot{G}$ is a signed multigraph.

We proceed by a concept of signed line graphs which is frequently used in spectral considerations: see [1, 4, 7, 9]; a different approach can be found in [11. For a signed graph $\dot{G}$, we introduce the vertex-edge orientation $\eta: V(\dot{G}) \times E(\dot{G}) \rightarrow$ $\{1,0,-1\}$ formed by obeying the following rules: (1) $\eta(i, j k)=0$ if $i \notin\{j, k\}$, (2) $\eta(i, i j)=1$ or $\eta(i, i j)=-1$, and $(3) \eta(i, i j) \eta(j, i j)=-\sigma(i j)$. In fact, each edge gets two orientations, so $\eta$ is also called a bi-orientation. The vertex-edge incidence matrix $B_{\eta}$ is the matrix whose rows and columns are indexed by $V(\dot{G})$ and $E(\dot{G})$ respectively, such that its $(i, e)$-entry is equal to $\eta(i, e)$. The following relation, valid also in the case that multiple edges exist, is well known:

$$
B_{\eta}^{\top} B_{\eta}=2 I+A_{L(\dot{G})},
$$

where $L(\dot{G})$ is taken to be the signed line graph of $\dot{G}$. A signed line graph defined in this way depends on orientation (in other words, it is not unique), but it is easy to show that different orientations produce switching equivalent signed line graphs. We also observe that this concept does not generalize the concept of line graphs. 
For example, the signed line graph of the positive triangle is the negative triangle, while the triangle (interpreted as a graph) is isomorphic to its line graph.

Since $B_{\eta}^{\top} B_{\eta}$ is positive semidefinite, we deduce that the spectrum of $L(\dot{G})$ is bounded (from below) by -2 . In analogy to the theory of unsigned graphs, we say that a connected signed graph is exceptional if its least eigenvalue is greater than or equal to -2 , but it is not a signed line graph. All the exceptional signed graphs whose least eigenvalue is greater than -2 are obtained in [4]. Up to switching isomorphism, there are exactly 1507 exceptional signed graphs with the above property; each of them has six, seven, or eight vertices.

We continue with star complements. The entire concept is transferred from the domain of graphs [3, 6]. Given a signed graph $\dot{G}$ with $n$ vertices, let $\mu$ be its eigenvalue and $P$ a matrix representing the orthogonal projection of $\mathbb{R}^{n}$ onto the eigenspace $\mathcal{E}(\mu)$ with respect to the canonical basis $\left\{\mathbf{e}_{\mathbf{1}}, \mathbf{e}_{\mathbf{2}}, \ldots, \mathbf{e}_{\mathbf{n}}\right\}$. The set $S \subseteq V(\dot{G})=\{1,2, \ldots, n\}$ such that the vectors $P \mathbf{e}_{i}(i \in S)$ make a basis of $\mathcal{E}(\mu)$ is called a star set for $\mu$. We use $\dot{S}$ to denote the signed graph induced by $S$. The signed graph induced by the set $V(\dot{G}) \backslash S$ is called a star complement for $\mu$. It follows that $\mu$ is not an eigenvalue of the star complement and $|S|$ is equal to the multiplicity of $\mu$ in $\dot{G}$. We use the notation $k=n-|S|$.

The following result is known as the Reconstruction Theorem.

Theorem 2.1 (cf. [3, Theorem 5.1.7]). Let $\dot{G}$ be a signed graph with adjacency matrix

$$
\left(\begin{array}{cc}
A_{\dot{S}} & B^{\top} \\
B & A_{\dot{H}}
\end{array}\right)
$$

where $A_{\dot{S}}$ is the adjacency matrix of the subgraph induced by a vertex set $S$, while $\dot{H}$ is the subgraph induced by $V(\dot{G}) \backslash S$. Then $S$ is a star set for $\mu$ if and only if $\mu$ is not an eigenvalue of $\dot{H}$ and

$$
\mu I-A_{\dot{S}}=B^{\top}\left(\mu I-A_{\dot{H}}\right)^{-1} B .
$$

This result was initially formulated in the context of graphs. Since every graph can be interpreted as a signed graph, it follows that Theorem 2.1 is an extension to the field of signed graphs.

If $\dot{H}$ is a star complement for an eigenvalue $\mu$ and $\mathbf{x}, \mathbf{y} \in \mathbb{R}^{k}$, we define the bilinear form

$$
\langle\mathbf{x}, \mathbf{y}\rangle=\mathbf{x}^{\top}\left(\mu I-A_{\dot{H}}\right)^{-1} \mathbf{y} .
$$

By virtue of Theorem 2.1 if $\mu$ is not an eigenvalue of $\dot{H}$ (with $k$ vertices), then there exists a signed graph $\dot{G}$ with $\dot{H}$ as a star complement for $\mu$ if and only if

$$
\left\langle\mathbf{b}_{i}, \mathbf{b}_{i}\right\rangle=\mu \quad \text { and } \quad\left\langle\mathbf{b}_{i}, \mathbf{b}_{j}\right\rangle \in\{1,0,-1\},
$$

for all distinct $i, j \in S$, where $\mathbf{b}_{i}$ and $\mathbf{b}_{j}$ determine neighbourhoods of $i$ and $j$ in $\dot{H}$, respectively.

The vectors $\mathbf{b}_{i}(1 \leq i \leq n-k)$ form the submatrix $B$ of 2.1 . Also, if $\left\langle\mathbf{b}_{i}, \mathbf{b}_{j}\right\rangle=0$ (resp. $\left\langle\mathbf{b}_{i}, \mathbf{b}_{j}\right\rangle=-1,\left\langle\mathbf{b}_{i}, \mathbf{b}_{j}\right\rangle=1$ ), then $i \nsim j$ (resp. $i \stackrel{+}{\sim} j, i 亢 j$ ). The matrix $\left(\mu I-A_{\dot{H}}\right)^{-1}$ can be computed by [3, Proposition 5.1.11]. 
Each of the mentioned 1507 exceptional signed graphs is a star complement for -2 in an (also) exceptional signed graph. Thus, we can say that exceptional signed graphs are characterized by means of their star complements. A similar characterization of exceptional graphs can be found in [3]. Here is another illustration.

Example 2.2. A triangle with positive edges is a star complement for -2 in the signed graph of Figure 1 (with spectrum $\left[2^{3},(-2)^{3}\right]$ ). Indeed, by using Theorem 2.1. we easily compute the matrix $B$ and the adjacency matrix of $\dot{S}$. Observe that the resulting signed graph is not exceptional.

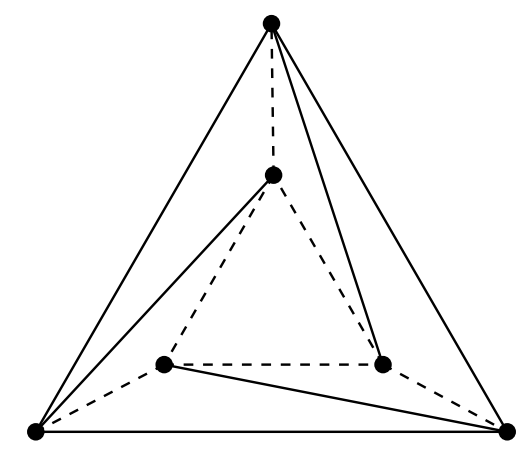

Figure 1. A signed graph for Example 2.2 (Negative edges are dashed.)

\section{Results}

We start with the following theorem.

Theorem 3.1. If $k K_{1}$ is a star complement for $\mu$ in a signed graph $\dot{G}$ with $n$ vertices and adjacency matrix 2.1) (for $A_{\dot{H}}=O$ ), then

(i) $B^{\top} B=\mu\left(\mu I-A_{\dot{S}}\right)$;

(ii) the $(n-k) \times(n-k)$ top-left block $X^{(p)}$ of $A_{\dot{G}}^{p}(p \geq 3)$ is determined by the recurrence

$$
\left\{\begin{array}{l}
X^{(p)}=X^{(p-1)} A_{\dot{S}}+\mu X^{(p-2)}\left(\mu I-A_{\dot{S}}\right) \\
X^{(1)}=A_{\dot{S}} \\
X^{(2)}=A_{\dot{S}}^{2}-\mu A_{\dot{S}}+\mu^{2} I
\end{array}\right.
$$

(iii) the number of eigenvalues of $\dot{S}$ does not exceed the number of eigenvalues of $\dot{G}$. 
Proof. (i): If $B=\left(\mathbf{b}_{1}\left|\mathbf{b}_{2}\right| \ldots \mid \mathbf{b}_{n-k}\right)$, then by 2.2 we have

$$
\left\langle\mathbf{b}_{i}, \mathbf{b}_{j}\right\rangle=\mathbf{b}_{i}^{\top} \frac{1}{\mu} I \mathbf{b}_{j}= \begin{cases}\mu, & \text { if } i=j ; \\ 0, & \text { if } i \nsim j ; \\ -1, & \text { if } i \sim j ; \\ 1, & \text { if } i \sim j .\end{cases}
$$

In other words, $B^{\top} B=\mu\left(\mu I-A_{\dot{S}}\right)$.

(ii): Considering the corresponding top-left block of $A_{\dot{G}}$, we immediately get $X^{(1)}=A_{\dot{S}}$ and $X^{(2)}=A_{\dot{S}}^{2}+B^{\top} B=A_{\dot{S}}^{2}-\mu A_{\dot{S}}+\mu^{2} I$. In addition, for $p \geq 3$, we have

$$
X^{(p)}=X^{(p-1)} A_{\dot{S}}+X^{(p-2)} B^{\top} B=X^{(p-1)} A_{\dot{S}}+\mu X^{(p-2)}\left(\mu I-A_{\dot{S}}\right),
$$

completing (3.1).

(iii): Assume that $\dot{G}$ has exactly $t$ eigenvalues. Since the top-left block of $A_{\dot{G}}^{p}$ is a polynomial in $A_{\dot{S}}$ of degree at most $p$ (by (3.1)), considering the minimal polynomial of $A_{\dot{G}}$ we deduce that there exists a polynomial $P$ of degree at most $t$ such that $P\left(A_{\dot{S}}\right)=O$, which concludes the proof.

Assume now that $\dot{G}$ has two eigenvalues, $\lambda$ and $\mu$. Computing its minimal polynomial, we get

$$
A_{\dot{G}}^{2}-(\lambda+\mu) A_{\dot{G}}+\lambda \mu I=O .
$$

It follows immediately that $\dot{G}$ is regular with vertex degree $-\lambda \mu$, which implies that $\lambda$ and $\mu$ are non-zero; in fact, exactly one of them is positive.

Lemma 3.2. If $k K_{1}$ is a star complement for $\mu$ in a signed graph $\dot{G}$ with $n$ vertices and two eigenvalues, then $2 k \leq n$.

Proof. If the adjacency matrix of $\dot{G}$ is given by (2.1), then using (3.3) we get $B B^{\boldsymbol{\top}}=-\lambda \mu I$, i.e., $\operatorname{rank}\left(B B^{\boldsymbol{\top}}\right)=k$ (as $\lambda$ and $\mu$ are non-zero). On the other hand, $\operatorname{rank}\left(B B^{\top}\right) \leq \operatorname{rank}(B) \leq n-k$, and the result follows.

By Theorem 3.1(iii), if $\dot{G}$ has two eigenvalues, then $\dot{S}$ has at most two eigenvalues. We consider them in more detail.

Theorem 3.3. Let a signed graph $\dot{G}$ with $n$ vertices and two eigenvalues, $\lambda$ and $\mu$, be decomposed into the star complement $k K_{1}$ for $\mu$ and the signed graph $\dot{S}$ induced by the star set. Then either

(i) $\dot{S}$ is isomorphic to $k K_{1}$ and $\lambda=-\mu$, or

(ii) $\dot{S}$ has two eigenvalues: $\lambda+\mu$ with multiplicity $k$ and $\mu$ with multiplicity $n-2 k$.

Proof. Let $\mathbf{e}_{i}(1 \leq i \leq k)$ denote the $i$ th vector of the canonical basis of $\mathbb{R}^{k}$. Observe that $B^{\top} \mathbf{x} \neq \mathbf{0}$ holds for every non-zero vector $\mathbf{x} \in \mathbb{R}^{k}$, as otherwise we would have $-\lambda \mu I \mathbf{x}=B B^{\top} \mathbf{x}=\mathbf{0}$, which is impossible.

By (3.3), we obtain $A_{\dot{S}} B^{\top}=(\lambda+\mu) B^{\top}$; hence,

$$
A_{\dot{S}} B^{\top} \mathbf{e}_{i}=(\lambda+\mu) B^{\top} \mathbf{e}_{i}
$$


i.e., $\lambda+\mu$ is an eigenvalue of $A_{\dot{S}}$. As the kernel of $B^{\top}$ trivially intersects the space $\mathbb{R}^{k}$, the multiplicity of $\lambda+\mu$ is at least $k$.

Denote by $\bar{\mu}$ the average value of the $n-2 k$ remaining eigenvalues (taken with their repetition). Considering $\operatorname{tr}\left(A_{\dot{G}}\right)=0$ and $\operatorname{tr}\left(A_{\dot{S}}\right)=0$, we get $k \lambda+(n-k) \mu=0$ and $k(\lambda+\mu)+(n-2 k) \bar{\mu}=0$, respectively. These equalities give $(n-2 k)(\mu-\bar{\mu})=0$. Hence, either $2 k=n$ or $\mu=\bar{\mu}$. In the former case, we immediately arrive at (i). In the latter, since $\dot{S}$ is a subgraph of $\dot{G}$, using the interlacing argument we get that $\mu$ is the unique remaining eigenvalue of $\dot{S}$ with multiplicity $n-2 k$; hence, (ii).

Observe that in Theorem 3.3 (i), $\dot{G}$ is bipartite and $k K_{1}$ is a star complement for both $\lambda$ and $\mu$. We proceed with a closer characterization. A matrix $M$ is said to be orthogonal if $M^{\top} M=M M^{\top}=r I$, for $r \neq 0$. (There is an equivalent approach with $r=1$.)

Corollary 3.4. Under the assumptions of Theorem 3.3. $\dot{S}$ is totally disconnected if and only if the matrix $B$ of (2.1) is orthogonal.

Proof. If $\dot{S}$ is totally disconnected, then by Theorem $3.3(\mathrm{i}), \lambda=-\mu$, which in conjunction with $(3.3)$ gives $B^{\top} B=B B^{\top}=-\lambda \mu I$.

Conversely, if $B$ is orthogonal, then it follows by Theorem 3.1 (i) that $\dot{S}$ is totally disconnected.

Let us take a break with an example illustrating the result of Theorem 3.3 (i).

Example 3.5. If $B$ is a Hadamard matrix or an adjacency matrix of a signed graph with two symmetric eigenvalues, then the signed graph $\dot{G}$ with adjacency matrix

$$
\left(\begin{array}{cc}
O & B^{\top} \\
B & O
\end{array}\right)
$$

has two symmetric eigenvalues and admits a decomposition into two isomorphic totally disconnected signed graphs. Each of them is a star complement for both eigenvalues of $\dot{G}$.

A particular case of Theorem 3.3(ii) is considered in the following subsection. Another particular case is treated in the forthcoming Subsection 3.2 .

3.1. Case: $\dot{G}$ has two eigenvalues, both $\geq-2$. If $\dot{G}$ is connected and its least eigenvalue is $\geq-2$, then $\dot{G}$ is either a signed line graph or an exceptional signed graph. An exceptional signed graph has a representation in the root system $E_{8}$, but not in any $D_{k}$. We presume that the reader is familiar with these and some related root systems (like $E_{7}$ or $E_{6}$ ), and also with the concept of their positive roots. In any case, all the necessary definitions can be found in [4, 7]. The following two results, taken respectively from [9] and [7], are needed.

Theorem $3.6([9])$. A connected signed line graph has two eigenvalues if and only if the corresponding signed root graph is switching equivalent to

(i) the star $K_{1, n-1}$, 
(ii) the negative quadrangle or the signed multigraph obtained by inserting the (parallel) negative edge between the vertices of degree 2 of the path with four vertices,

(iii) the complete graph $K_{n}$, or

(iv) a connected signed doubled regular graph with $n$ vertices, in all cases, for $n \geq 3$.

Obviously, a signed root graph $K_{1, n-1}$ gives a signed line graph that is switching equivalent to $K_{n}$. Both signed root graphs of Theorem 3.6 (ii) give the negative quadrangle whose spectrum is $\left[\sqrt{2}^{2},-\sqrt{2}^{2}\right]$. Using $(3.3)$, we compute the spectra of the signed line graphs of Theorem 3.6 (iii) \& (iv); they are given by

$$
L\left(K_{n}\right):\left[(n-2)^{n-1},(-2)^{\left(\begin{array}{c}
n-1 \\
2
\end{array}\right)}\right], \quad L(\ddot{F}):\left[2(r-1)^{n},(-2)^{n(r-1)}\right],
$$

where $F$ is a regular graph with $n$ vertices and degree $r$.

Theorem 3.7 ([7]). If $\dot{G}$ is an exceptional signed graph with $n$ vertices, then $n \geq 6$, the multiplicity of -2 in its spectrum is $n-k$, for some $k \in\{6,7,8\}$, and $\dot{G}$ is an induced subgraph of a signed graph represented by all the positive roots of $E_{k}$.

By [7], for $6 \leq k \leq 8$, the signed graph represented by all the positive roots of $E_{k}$ is unique (up to the switching isomorphism). Denote it by $\dot{M}_{k}$. By the same reference (and this can also be computed directly), its spectrum is given by

$$
\dot{M}_{6}:\left[10^{6},(-2)^{30}\right], \quad \dot{M}_{7}:\left[16^{7},(-2)^{56}\right], \quad \dot{M}_{8}:\left[28^{8},(-2)^{112}\right] \text {. }
$$

We proceed with the following result concerning star complements in signed line graphs.

Theorem 3.8. The following statements hold (in all cases $n$ is assumed to be at least 3$)$ :

(i) $K_{1}$ is the unique star complement for -1 in $K_{n} . K_{n}$ does not have a totally disconnected star complement for $n-1$.

(ii) $2 K_{1}$ is the unique star complement for both eigenvalues of a negative quadrangle.

(iii) $L\left(K_{1, n-1}\right)$ does not have a totally disconnected star complement.

(iv) If $F$ is a connected $r$-regular signed graph, then $L(\ddot{F})$ has a totally disconnected star complement if and only if $F$ has a perfect matching. Such a star complement is induced by a perfect matching and corresponds to -2 ; if $r=2$, it simultaneously corresponds to the other eigenvalue of $L(\ddot{F})$.

Proof. (i), (ii): This result follows directly.

(iii): A totally disconnected subgraph of $L\left(K_{n}\right)$ is induced by a matching in $K_{n}$. If such a graph is a star complement, then for -2 the matching must count $n-1$ edges, and for $n-2$ it must count $\left(\begin{array}{c}n-1 \\ 2\end{array}\right)$ edges. Both are impossible (under our assumption on $n$ ), as a matching in $K_{n}$ has at most $\left\lfloor\frac{n}{2}\right\rfloor$ edges.

(iv): Similarly, a totally disconnected star complement in $L(\ddot{F})$ is induced by a matching in $\ddot{F}$. For the eigenvalue -2 , such a matching must count $n$ edges, which occurs only if $F$ has a perfect matching. For the eigenvalue $2(r-1)$, the size of a 
matching in $\ddot{F}$ must attain $n(r-1)$, which occurs only if $r=2$ and $F$ has a perfect matching, and we are done. The opposite implication is verified directly.

We continue with star complements in exceptional signed graphs.

Theorem 3.9. If an exceptional signed graph $\dot{G}$ with two eigenvalues has a totally disconnected star complement, then its spectrum is $\left[\sqrt{3}^{4},-\sqrt{3}^{4}\right],\left[2 i^{7},(-2)^{7 i}\right]$, for $1 \leq i \leq 8$, or $\left[i^{8},(-2)^{4 i}\right]$, for $2 \leq i \leq 28$.

For the first spectrum, $i=1$ in the second and $i=2$ in the third, the star complement corresponds to both eigenvalues. In the remaining cases, it corresponds to -2 .

Proof. By Theorem 3.7 -2 is an eigenvalue of a signed graph whenever it has at least nine vertices. Considering those with fewer than nine vertices (they can be found in [4]), we arrive at the unique solution with spectrum $\left[\sqrt{3}^{4},-\sqrt{3}^{4}\right]$.

Assume further that -2 is an eigenvalue of $\dot{G}$ and denote the other eigenvalue by $\lambda$. Consider totally disconnected star complements for -2 . By Theorem 3.7 the multiplicity of $\lambda$ is $k(6 \leq k \leq 8)$ and $\dot{G}$ is an induced subgraph of $\dot{M}_{k}$. The corresponding star complement is $k K_{1}$.

The case $k=6$ cannot occur, as the size of the largest co-clique in $\dot{M}_{6}$ is 4 , and so the size of the largest co-clique in $\dot{G}$ is at most 4 . In the remaining cases the size of the largest co-clique in $\dot{M}_{k}$ is equal to $k$. Considering $\operatorname{tr}\left(A_{\dot{G}}\right)=0$ and $\operatorname{tr}\left(A_{\dot{G}}^{2}\right)=n$ ( $n$ being the number of vertices in $\dot{G}$ ), we get $n=\frac{k}{2}(\lambda+2)$. Since $n$ is an integer and is bounded by the number of vertices in $\dot{M}_{k}$, we arrive at the desired spectra.

The remainder of the proof, concerning star complements for $\lambda$, follows by Theorem 3.3

Example 3.10. We confirm the existence of $\dot{G}$ with the second spectrum of Theorem 3.9 for every $i$ and for the third spectrum for every even $i$, by a successive removal of a selected co-clique. Precisely, we start with $\dot{M}_{k}(k \in\{7,8\})$, then remove the roots that correspond to a selected co-clique of size $k$, and check whether the signed graph obtained contains a co-clique of the same size. Then we continue with this signed graph in the role of $\dot{M}_{k}$.

3.2. Case: $k=\mu^{2}$. Here, by Theorem 3.1(i), all the entries of $B$ are \pm 1 s. We consider the case where $\dot{G}$ is decomposed into the star complement $\mu^{2} K_{1}$ and either a totally disconnected graph or a signed graph which is switching equivalent to a complete graph. Note that, by $(3.2),\left\langle\mathbf{b}_{i}, \mathbf{b}_{j}\right\rangle=0$ cannot occur if $\mu^{2}$ is odd. In other words, for $\dot{S}$ totally disconnected, $\mu^{2}$ must be even.

Theorem 3.11. If a signed graph $\dot{G}$ with $n$ vertices is decomposed into the star complement $\mu^{2} K_{1}$ (for $\mu$ ) and a signed graph $\dot{S}$, then $n \leq 2 \mu^{2}$ and

(i) if $\dot{S}$ is totally disconnected, the spectrum of $\dot{G}$ is

$$
\left[\mu^{n-\mu^{2}}, 0^{2 \mu^{2}-n},(-\mu)^{n-\mu^{2}}\right]
$$


(ii) if $\dot{S}$ is switching equivalent to a complete graph, the spectrum of $\dot{G}$ is

$$
\left[\mu^{n-\mu^{2}}, n-\mu^{2}-\mu-1,0^{2 \mu^{2}-n},(-\mu-1)^{n-\mu^{2}-1}\right] .
$$

Proof. The inequality $n \leq 2 \mu^{2}$ follows by modifying the proof of Lemma 3.2 , that is, by interchanging $\mu^{2} \bar{K}_{1}$ and $\dot{S}$. Indeed, since $B^{\top} B=\mu\left(\mu I-A_{\dot{S}}\right)$ (by Theorem $3.1(\mathrm{i}))$ and $\dot{S}$ is either totally disconnected or complete, we have $\operatorname{rank}\left(B^{\top} B\right)=$ $n-\mu^{2}$, and the result follows from $\operatorname{rank}\left(B^{\top} B\right) \leq \operatorname{rank}(B) \leq \mu^{2}$.

Computing the characteristic polynomial of $\dot{G}$, we get

$$
\begin{aligned}
\left|x I-A_{\dot{G}}\right| & =\left|x I-\left(\begin{array}{cc}
A_{\dot{S}} & B^{\boldsymbol{\top}} \\
B & O
\end{array}\right)\right|=\left|\begin{array}{cc}
x I_{n-\mu^{2}}-A_{\dot{S}} & -B^{\boldsymbol{\top}} \\
-B & x I_{\mu^{2}}
\end{array}\right| \\
& =\left|x I_{\mu^{2}}\right| \cdot\left|x I_{n-\mu^{2}}-A_{\dot{S}}-B^{\boldsymbol{\top}}\left(x I_{\mu^{2}}\right)^{-1} B\right| \\
& =x^{\mu^{2}}\left|x I_{n-\mu^{2}}-A_{\dot{S}}-\frac{1}{x} \mu\left(\mu I_{n-\mu^{2}}-A_{\dot{S}}\right)\right| \\
& =x^{\mu^{2}}\left|\frac{x^{2}-\mu^{2}}{x} I_{n-\mu^{2}}-\frac{x-\mu}{x} A_{\dot{S}}\right| \\
& =x^{2 \mu^{2}-n}\left|\left(x^{2}-\mu^{2}\right) I_{n-\mu^{2}}-(x-\mu) A_{\dot{S}}\right|,
\end{aligned}
$$

where the equality in the second line follows by a standard technique for computing determinants; see, for example, [2, Lemma 2.2]. Now, for (i) we have $A_{\dot{S}}=O_{n-\mu^{2}}$, while for (ii) we have $A_{\dot{S}}=A_{K_{n-\mu^{2}}}=J_{n-\mu^{2}}-I_{n-\mu^{2}}$. In both cases the desired spectrum follows easily.

The signed graphs of Example 3.5 are also examples for Theorem 3.11(i). Some others can easily be constructed by hand. We conclude the paper with an example illustrating the result of Theorem 3.11 (ii).

Example 3.12. If $\dot{G}$ is a signed graph of Theorem 3.11 (ii), then due to switching equivalence we may assume that the complete graph stands for $\dot{S}$. In this case, for a fixed $i \in V\left(K_{n-\mu^{2}}\right)$, the column $\mathbf{b}_{i}$ of $B$ may be taken to be all-1, while for every other $j \in V\left(K_{n-\mu^{2}}\right), \mathbf{b}_{j}$ contains exactly $\frac{\mu(\mu-1)}{2}$ ones (due to (3.2)). If so, then the vertices of $K_{n-\mu^{2}}$ distinct from the fixed one may be considered as points and the vertices of (the star complement) $\mu^{2} K_{1}$ as blocks of a block design in which a point is arranged into a block precisely if the corresponding vertices are adjacent by a positive edge. In our case, every point is arranged into $\frac{\mu(\mu-1)}{2}$ blocks; to avoid trivial cases, we further assume that $\mu(\mu-1) \neq 0$.

If every block contains $r$ points, and every pair of points is simultaneously contained in $l$ blocks, then the block design is recognized as a balanced incomplete block design (so-called BIBD) with parameters $\left(n-\mu^{2}-1, r, l\right)$. Clearly, in our case $l=0$, by $(3.2)$. Using the relations between the parameters $\mu^{2} r=\frac{\mu(\mu-1)}{2}\left(n-\mu^{2}-1\right)$ and $\frac{\mu(\mu-1)}{2}(r-1)=0$ [8, $\left.\S 3.8 .1\right]$, we get $n-\mu^{2}-1=\frac{2 \mu^{2}}{\mu(\mu-1)}$ and $r=1$.

Therefore, a BIBD with parameters $\left(\frac{2 \mu^{2}}{\mu(\mu-1)}, 1,0\right)$ gives our $\dot{G}$. For example, by setting $\mu=3$, we arrive at $n=13$ and 


$$
B^{\top}=\left(\begin{array}{ccccccccc}
-1 & 1 & -1 & -1 & -1 & -1 & 1 & 1 & -1 \\
1 & -1 & -1 & -1 & -1 & 1 & -1 & -1 & 1 \\
-1 & -1 & 1 & 1 & 1 & -1 & -1 & -1 & -1 \\
1 & 1 & 1 & 1 & 1 & 1 & 1 & 1 & 1
\end{array}\right)
$$

giving $\dot{G}$ with spectrum $\left[3^{4}, 0^{6},(-4)^{3}\right]$.

\section{REFERENCES}

[1] P. J. Cameron et al., Line graphs, root systems, and elliptic geometry, J. Algebra 43 (1976), no. 1, 305-327. MR 0441787

[2] D. Cvetković, M. Doob and H. Sachs, Spectra of graphs, third edition, Johann Ambrosius Barth, Heidelberg, 1995. MR 1324340

[3] D. Cvetković, P. Rowlinson and S. Simić, Spectral generalizations of line graphs: On graphs with least eigenvalue -2, London Mathematical Society Lecture Note Series, 314, Cambridge University Press, Cambridge, 2004. MR 2120511

[4] G. Greaves et al., Edge-signed graphs with smallest eigenvalue greater than -2, J. Combin. Theory Ser. B 110 (2015), 90-111. MR 3279389.

[5] W. H. Haemers and D. G. Higman, Strongly regular graphs with strongly regular decomposition, Linear Algebra Appl. 114/115 (1989), 379-398. MR 0986885

[6] P. Rowlinson, Star complements in finite graphs: a survey, Rend. Sem. Mat. Messina Ser. II 8(24) (2001/02), suppl., 145-162. MR 1964838

[7] Z. Stanić, Notes on exceptional signed graphs, Ars Math. Contemp. 18 (2020), no. 1, 105-115. MR 4154730

[8] Z. Stanić, Regular graphs: a spectral approach, De Gruyter Series in Discrete Mathematics and Applications, 4, De Gruyter, Berlin, 2017. MR 3753662

[9] Z. Stanić, Spectra of signed graphs with two eigenvalues, Appl. Math. Comput. 364 (2020), 124627, 9 pp. MR 3996372 .

[10] Z. Stanić, Unions of a clique and a co-clique as star complements for non-main graph eigenvalues, Electron. J. Linear Algebra 35 (2019), 90-99. MR 3939834.

[11] T. Zaslavsky, Matrices in the theory of signed simple graphs, in Advances in discrete mathematics and applications: Mysore, 2008, 207-229, Ramanujan Math. Soc. Lect. Notes Ser., 13, Ramanujan Math. Soc., Mysore. MR 2766941

\section{Zoran Stanić}

Faculty of Mathematics, University of Belgrade, Studentski trg 16, 11000 Belgrade, Serbia zstanic@math.rs

Received: March 3, 2019

Accepted: December 17, 2019 